\title{
Stability analysis of Uzawa-Lucas endogenous growth model
}

\author{
William A. Barnett • Taniya Ghosh
}

Received: 17 November 2013 / Accepted: 20 November 2013 / Published online: 10 December 2013 (C) SAET 2013

\begin{abstract}
This paper analyzes, within its feasible parameter space, the dynamics of the Uzawa-Lucas endogenous growth model. The model is solved from a centralized social planner perspective as well as in the model's decentralized market economy form. We examine the stability properties of both versions of the model and locate Hopf and transcritical bifurcation boundaries. In an extended analysis, we investigate the existence of Andronov-Hopf bifurcation, branch point bifurcation, limit point cycle bifurcation, and period-doubling bifurcations. While these all are local bifurcations, the presence of global bifurcation is confirmed as well. We find evidence that the model could produce chaotic dynamics, but our analysis cannot confirm that conjecture. It is important to recognize that bifurcation boundaries do not necessarily separate stable from unstable solution domains. Bifurcation boundaries can separate one kind of unstable dynamics domain from another kind of unstable dynamics domain, or one kind of stable dynamics domain from another kind (called soft bifurcation), such as bifurcation from monotonic stability to damped periodic stability or from damped periodic to damped multi-periodic stability. While there are an infinite number of kinds of unstable dynamics, some very close to stability in appearance, there also are an infinite number of kinds of stable dynamics. Hence, subjective prior views on whether the economy is or is not stable provide little guidance without mathematical analysis of model dynamics. When a bifurcation boundary crosses the parameter estimates'
\end{abstract}

\footnotetext{
W. A. Barnett ( $\square)$

University of Kansas, Lawrence, USA

e-mail: barnett@ku.edu; williamabarnett@gmail.com

W. A. Barnett

Center for Financial Stability, NY City, USA

T. Ghosh

Indira Gandhi Institute of Development Research (IGIDR), Reserve Bank of India, Mumbai, India
} 
confidence region, robustness of dynamical inferences from policy simulations are compromised, when conducted in the usual manner only at the parameters' point estimates.

Keywords Bifurcation - Endogenous growth - Lucas-Uzawa model · Hopf · Inference robustness · Dynamics · Stability

JEL Classification C5 $\cdot$ C6 $\cdot$ E3 $\cdot$ E61

\section{Introduction}

The Uzawa-Lucas model [(Uzawa (1965) and Lucas (1988)], upon which many others have been built, is among the most important endogenous growth models. The model has two sectors: the human capital production sector and the physical capital production sector, producing human capital and physical capital, respectively. Individuals have the same level of work qualification and expertise $(H)$. They allocate some of their time toward producing final goods and dedicate the remaining time to training and studying.

The social planner solution for the Uzawa-Lucas model is saddle path stable, but the representative agent's equilibrium can be indeterminate, as shown by Benhabib and Perli (1994). As a result of the presence of externalities in human capital, the market solution is different from the social planner solution. The externality creates a distinction between return on capital, as perceived by the representative agent, to that perceived by a social planner.

In this paper we provide a detailed bifurcation analysis of the model. Our task is to examine whether the dynamics of the model changes within the feasible parameter space of the model. A system undergoes a bifurcation, if a small smooth change in a parameter produces a sudden qualitative or topological change in the nature of singular points and trajectories of the system. The presence of bifurcation damages the inference robustness of the dynamics, when inferences are based on point estimates of the model. Hence, knowing the stability boundaries within the feasible region of the parameter space, especially near the point estimates, can lead to more robust dynamical inferences and more reliable policy conclusions.

Using Mathematica, we locate transcritical and Hopf bifurcation boundaries in two-dimension and three-dimension diagrams. The numerical continuation package, Matcont, is used to analyze further the stability properties of the limit cycles generated by Hopf bifurcations and the presence of other kinds of bifurcations. We show the existence of Hopf, branch-point, limit-point-of-cycles, and period-doubling bifurcations within the feasible parameters set of the model's parameter space. While these are all local bifurcations, presence of global bifurcation is also confirmed. There is some evidence of the possibility of chaotic dynamics through the detected series of period-doubling bifurcations, known to converge to chaos. Some of these results have not previously been demonstrated in the literature on endogenous growth models.

Benhabib and Perli (1994) analyzed the stability property of the long-run equilibrium in the Lucas (1988) model. They have also detected a Hopf bifurcation in the 
Lucas (1988) model. Arnold (2000a; 2000b) analyzed the stability of equilibrium in the Romer (1990) model. Arnold (2006) has done the same for the Jones (1995) model. Mondal (2008) examined the dynamics of the Grossman and Helpman (1991b) model of endogenous product cycles. The results derived in those papers provide important insights to researchers considering different policies. But, as in the Benhabib and Perli (1994) paper, a detailed bifurcation analysis has not been provided so far for many of these popular endogenous growth models. The current paper aims to fill this gap for the Uzawa-Lucas model.

As pointed out by Banerjee et al. (2011): "Just as it is important to know for what parameter values a system is stable or unstable, it is equally important to know the nature of stability (e.g. monotonic convergence, damped single periodic convergence, or damped multi-periodic convergence) or instability (periodic, multi-periodic, or chaotic)." Barnett and his coauthors have made significant contribution in this area. Barnett and He $(1999 ; 2002)$ examined the dynamics of the Bergstrom-Wymer continuous-time dynamic macroeconometric model of the UK economy. Both transcritical bifurcation boundaries and the Hopf bifurcation boundaries for the model were found. Barnett and He (2008) have found singularity bifurcation boundaries within the parameter space for the Leeper and Sims (1994) model. Barnett and Duzhak (2010) found Hopf and period-doubling bifurcations in a New Keynesian model. More recently, Banerjee et al. (2011) examined the possibility of cyclical behavior in the Marshallian macroeconomic model and Barnett and Eryilmaz (2013a; 2013b) have found bifurcation in open economy models, while Barnett and Ghosh (2013) have found bifurcation boundaries within a variant of the Jones (1995) semi-endogenous growth model.

\section{The Uzawa-Lucas model}

The production function in the physical sector is given by $Y=A K^{\alpha}(\varepsilon h L)^{1-\alpha} h_{a}^{\zeta}, 0<$ $\alpha<1$, where $Y$ is output, $A$ is constant technology level, $K$ is physical capital, $\alpha$ is the share of physical capital, $L$ is labor, and $h$ is human capital per person. In addition, $\varepsilon$ and $(1-\varepsilon)$ are the fraction of labor time devoted to producing output and human capital, respectively, where $0<\varepsilon<1$. The quantity of labor, $\varepsilon h L$, is measured in efficiency units, employed to produce output, and $h_{\mathrm{a}}^{\zeta}$ measures the externality associated with average human capital of the work force, $h_{\mathrm{a}}$, where $\zeta$ is the positive externality parameter in the production of human capital. The physical capital accumulation equation in per capita terms is, $\dot{k}=A K^{\alpha}(\varepsilon h)^{1-\alpha} h_{\mathrm{a}}^{\varsigma}-c-(n+\delta) k$, and the human capital accumulation equation is $\dot{h}=\eta h(1-\varepsilon)$, where $\eta$ is defined as schooling productivity. The decision problem is

$$
\begin{aligned}
& \max _{c_{t}, \varepsilon_{t}} \int_{t}^{\infty} e^{-(\rho-n) t}\left[c(\tau)^{1-\sigma}-1\right] /(1-\sigma) d t \\
& \text { subject to } \dot{k}=A K^{\alpha}(\varepsilon h)^{1-\alpha} h_{a}^{\varsigma}-c-(n+\delta) k
\end{aligned}
$$

and, 


$$
\dot{h}=\eta(1-\varepsilon) h
$$

where $\rho(\rho>n>0)$ is the subjective discount rate, and $\sigma(\geq 0)$ is the inverse of the intertemporal elasticity of substitution in consumption.

\subsection{Social planner problem}

In solving the maximization problem, (1), subject to the physical capital accumulation Eq. 2 and the human capital accumulation Eq. 3, the social planner takes into account the externality associated with human capital. From the first-order conditions (see Appendix 2), we derive the equations describing the economy of the Uzawa-Lucas model from a social planner's perspective:

$$
\begin{aligned}
& \frac{\dot{k}}{k}=A k^{\alpha-1} \varepsilon^{1-\alpha} h^{1-\alpha+\zeta}-\frac{c}{k}-(n+\delta) ; \frac{\dot{h}}{h}=\eta(1-\varepsilon) ; \\
& \frac{\dot{c}}{c}=\frac{\alpha A k^{\alpha-1} \varepsilon^{1-\alpha} h^{1-\alpha+\zeta}-(\rho+\delta)}{\sigma} ; \\
& \frac{\dot{\varepsilon}}{\varepsilon}=\eta \frac{(1-\alpha+\zeta)}{(1-\alpha)} \varepsilon+\eta \frac{(1-\alpha+\zeta)}{\alpha}-\frac{c}{k}+\frac{(1-\alpha)}{\alpha}(n+\delta) ; \frac{\dot{L}}{L}=n .
\end{aligned}
$$

Let $m=\frac{Y}{K}$ and $g=\frac{c}{k}$. Taking logarithms of $m$ and $g$ and differentiating with respect to time, Eqs. 4 and 5 define the dynamics of Uzawa-Lucas model

$$
\begin{aligned}
& \frac{\dot{m}}{m}=-(1-\alpha) m+\frac{(1-\alpha)}{\alpha}(n+\delta)+\eta \frac{(1-\alpha+\zeta)}{\alpha} \\
& \frac{\dot{g}}{g}=\left(\frac{\alpha}{\sigma}-1\right) m-\frac{\rho}{\sigma}-\delta\left(\frac{1}{\sigma}-1\right)+g+n
\end{aligned}
$$

The steady state $\left(m^{*}, g^{*}\right)$ is given by $\dot{m}=\dot{g}=0$ and is derived to be

$$
\begin{aligned}
& m^{*}=\eta \frac{(1-\alpha+\zeta)}{\alpha}+\frac{(n+\delta)}{\alpha}, \\
& g^{*}=\frac{\rho-n}{\sigma}+\frac{(1-\alpha)}{\alpha}(n+\delta)+\eta \frac{(1-\alpha+\zeta)}{\alpha(1-\alpha)} \frac{(\sigma-\alpha)}{\sigma} .
\end{aligned}
$$

A unique steady state exists, if $\Lambda=\frac{(1-\alpha+\zeta)}{\alpha}(\sigma-1) \eta(1-\varepsilon)+\rho>0$. In addition, $\Lambda$ provides the necessary and sufficient condition for the transversality condition to hold for the consumer's utility maximization problem (see Appendix 1). Following the approach of Barro and Sala-i-Martin (2003) and Mattana (2004), it can be shown that social planner solution is saddle path stable. We linearize around the steady state, $s^{*}=\left(m^{*}, g^{*}\right)$, to analyze the local stability properties of the system defined by Eqs. 4 and 5 . The result is 


$$
\left[\begin{array}{c}
\dot{m} \\
\dot{g}
\end{array}\right]=\underbrace{\left[\begin{array}{ll}
\left.\frac{\partial \dot{m}}{\partial m}\right|_{s^{*}} & \left.\frac{\partial \dot{m}}{\partial g}\right|_{s^{*}} \\
\left.\frac{\partial \dot{g}}{\partial m}\right|_{s^{*}} & \left.\frac{\partial \dot{g}}{\partial g}\right|_{s^{*}}
\end{array}\right]}_{J_{s}}\left[\begin{array}{c}
m_{t}-m^{*} \\
g_{t}-g^{*}
\end{array}\right], \text { Where } J_{s}=\left[\begin{array}{cc}
-(1-\alpha) m^{*} & 0 \\
\left(\frac{\alpha}{\sigma}-1\right) g^{*} & g^{*}
\end{array}\right]
$$

As $m^{*}, g^{*}>0$, it follows that $\operatorname{Det}(J)=-(1-\alpha) m^{*} g^{*}<0$, hence saddle path stable.

\subsection{Representative agent problem}

From the first-order conditions (see Appendix 3) and setting $h=h_{\mathrm{a}}$, we derive the equations describing the economy of the Uzawa-Lucas model from a decentralized economy's perspective:

$$
\begin{aligned}
& \frac{\dot{k}}{k}=A k^{\alpha-1} \varepsilon^{1-\alpha} h^{1-\alpha+\zeta}-\frac{c}{k}-(n+\delta) ; \\
& \frac{\dot{h}}{h}=\eta(1-\varepsilon) ; \frac{\dot{c}}{c}=\frac{\alpha A k^{\alpha-1} \varepsilon^{1-\alpha} h^{1-\alpha+\zeta}-(\rho+\delta)}{\sigma} ; \\
& \frac{\dot{\varepsilon}}{\varepsilon}=\eta \frac{(\alpha-\zeta)}{\alpha} \varepsilon+\eta \frac{(1-\alpha+\zeta)}{\alpha}-\frac{c}{k}+\frac{(1-\alpha)}{\alpha}(n+\delta) ; \frac{\dot{L}}{L}=n .
\end{aligned}
$$

Let $m=\frac{Y}{K}$ and $g=\frac{c}{k}$. Taking logarithms of $m$ and $g$ and differentiating with respect to time, the following three equations define the dynamics of Uzawa-Lucas model

$$
\begin{aligned}
& \frac{\dot{m}}{m}=-(1-\alpha) m+\frac{(1-\alpha)}{\alpha}(n+\delta)+\eta \frac{(1-\alpha+\zeta)}{\alpha}-\eta \frac{\zeta}{\alpha} \varepsilon \\
& \frac{\dot{g}}{g}=\left(\frac{\alpha}{\sigma}-1\right) m-\frac{\rho}{\sigma}-\delta\left(\frac{1}{\sigma}-1\right)+g+n \\
& \frac{\dot{\varepsilon}}{\varepsilon}=\eta \frac{(\alpha-\zeta)}{\alpha} \varepsilon+\eta \frac{(1-\alpha+\zeta)}{\alpha}-g+\frac{(1-\alpha)}{\alpha}(n+\delta)
\end{aligned}
$$

The steady state $\left(m^{*}, g^{*}, \varepsilon^{*}\right)$ is given by $\dot{m}=\dot{g}=\dot{\varepsilon}=0$ and derived to be

$$
\begin{aligned}
& \varepsilon^{*}=1-\frac{(1-\alpha)(\rho-n-\eta)}{\eta[\zeta-\sigma(1-\alpha+\zeta)]}, \quad m^{*}=\eta \frac{\left[1-\alpha+\zeta\left(1-\varepsilon^{*}\right)\right]}{\alpha(1-\alpha)}+\frac{n}{\alpha}, \\
& g^{*}=\eta \frac{\left[1-\alpha+\zeta\left(1-\varepsilon^{*}\right)+\alpha \varepsilon^{*}\right]}{\alpha}+\frac{n(1-\alpha)}{\alpha} .
\end{aligned}
$$

Note that as shown by Benhabib and Perli (1994), $0<\frac{(1-\alpha)(\rho-n-\eta)}{\eta[\zeta-\sigma(1-\alpha+\zeta)]}<1$ is necessary for $0<\varepsilon^{*}<1$. A unique steady state exists if $\Lambda=\frac{(1-\alpha+\zeta)}{\alpha}(\sigma-1) \eta(1-\varepsilon)+$ $\rho>0$ and $0<\varepsilon<1$. 
$\Lambda$ is necessary and sufficient for the transversality condition to hold for the consumer's utility maximization problem (Appendix 1); and $0<\varepsilon^{*}<1$ is necessary for $m^{*}, g^{*}>0$. We linearize the system given by equations 6,7 and 8 around the steady state, $s^{*}=\left(m^{*}, g^{*}, \varepsilon^{*}\right)$, to acquire

$$
\begin{aligned}
& {\left[\begin{array}{c}
\dot{m} \\
\dot{g} \\
\dot{\varepsilon}
\end{array}\right]=\underbrace{\left[\begin{array}{lll}
\left.\frac{\partial \dot{m}}{\partial m}\right|_{s^{*}} & \left.\frac{\partial \dot{m}}{\partial g}\right|_{s^{*}} & \left.\frac{\partial \dot{m}}{\partial \varepsilon}\right|_{s^{*}} \\
\left.\frac{\partial \dot{g}}{\partial m}\right|_{s^{*}} & \left.\frac{\partial \dot{g}}{\partial g}\right|_{s^{*}} & \left.\frac{\partial \dot{g}}{\partial \varepsilon}\right|_{s^{*}} \\
\left.\frac{\partial \dot{\varepsilon}}{\partial m}\right|_{s^{*}} & \left.\frac{\partial \dot{\varepsilon}}{\partial g}\right|_{s^{*}} & \left.\frac{\partial \dot{\varepsilon}}{\partial \varepsilon}\right|_{s^{*}}
\end{array}\right]}_{J_{m}}\left[\begin{array}{l}
m_{t}-m^{*} \\
g_{t}-g^{*} \\
\varepsilon_{t}-\varepsilon^{*}
\end{array}\right] \text {, where, }} \\
& J_{m}=\left[\begin{array}{lll}
-(1-\alpha) m^{*} & 0 & -\eta \frac{\zeta}{\alpha} m^{*} \\
\left(\frac{\alpha}{\sigma}-1\right) g^{*} & g^{*} & 0 \\
0 & -\varepsilon^{*} & \eta \frac{(\alpha-\zeta)}{\alpha} \varepsilon^{*}
\end{array}\right]
\end{aligned}
$$

The characteristic equation associated with $J_{m}$ is $q^{3}+c_{2} q^{2}+c_{1} q+c_{0}=0$, where

$$
\begin{aligned}
& c_{0}=\eta \frac{[\sigma(1-\alpha+\zeta)-\zeta]}{\sigma} m^{*} g^{*} \varepsilon^{*}, c_{1}=\eta^{2} \frac{(\alpha-\zeta)}{\alpha} \varepsilon^{* 2}-(1-\alpha) m^{*} g^{*}, \\
& c_{2}=-\eta \frac{(2 \alpha-\zeta)}{\alpha} \varepsilon^{*} .
\end{aligned}
$$

\section{Bifurcation analysis of Uzawa-Lucas model}

In this section, we examine the existence of codimension 1 and 2, transcritical, and Hopf bifurcations in the system given by equations 6,7 , and 8 . The codimension, as defined by Kuznetsov (1998), is the number of independent conditions determining the bifurcation boundary. Varying a single parameter helps to identify codimension-1 bifurcation, and varying 2 parameters helps to identify codimension- 2 bifurcation.

An equilibrium point, $s^{*}$, of the system is called hyperbolic, if the coefficient matrix, $J_{m}$, has no eigenvalues with zero real parts. For small perturbations of parameters, there are no structural changes in the stability of a hyperbolic equilibrium, provided that the perturbations are sufficiently small. Therefore, bifurcations occur at nonhyperbolic equilibria only. A transcritical bifurcation occurs, when a system has a nonhyperbolic equilibrium at the bifurcation point with a geometrically simple zero eigenvalue. Also additional transversality conditions must be satisfied, as given by Sotomayor's Theorem [Barnett and He (1999)]. The first condition we are going to use to find the bifurcation boundary is $c_{0}=\operatorname{det}\left(J_{m}\right)=0$. The result is the following.

Theorem $1 J_{m}$ has zero eigenvalues, if

$$
\eta \frac{[\sigma(1-\alpha+\zeta)-\zeta]}{\sigma} m^{*} g^{*} \varepsilon^{*}=0
$$


Hopf bifurcations occur at points at which the system has a nonhyperbolic equilibrium with a pair of purely imaginary eigenvalues, but without zero eigenvalues. Also additional transversality conditions must be satisfied. We use the following theorem, based upon the version of the Hopf Bifurcation Theorem in Guckenheimer and Holmes (1983), to find that $J_{m}$ has precisely one pair of pure imaginary eigenvalues, if $c_{0}-c_{1} c_{2}=0$ and $c_{1}>0$. If $c_{0}-c_{1} c_{2} \neq 0$ and $c_{1}>0$, then $J_{m}$ has no pure imaginary eigenvalues. The result is:

Theorem 2 The matrix $J_{m}$ has precisely one pair of pure imaginary eigenvalues, if

$$
\left.\begin{array}{l}
\alpha m^{*} g^{*}((\alpha-1) \alpha \sigma+\zeta(\sigma-\alpha))+\eta^{2} \sigma \varepsilon^{* 2}(2 \alpha-\zeta)(\alpha-\zeta)=0 \text { and }, \\
\frac{\eta^{2}}{\alpha} \varepsilon^{* 2}(\alpha-\zeta)-(1-\alpha) m^{*} g^{*}>0
\end{array}\right\}
$$

Let $\vartheta^{*}=\{\eta, \zeta, \alpha, \rho, \sigma, n, \delta\}=(0.05,0.1,0.65,0.0505,0.15,0,0), \omega^{*}=\{\eta, \zeta, \alpha$, $\rho, \sigma, n, \delta\}=(0.05,0.1,0.75,0.0505,0.15,0,0)$. In the rest of the analysis, we locate the bifurcation boundaries by keeping some parameters free and setting the others fixed at $\vartheta^{*}$ or at $\omega^{*}$. These values are based on Benhabib and Perli (1994). We first locate the transcritical bifurcation boundaries and Hopf bifurcation boundaries given by Eqs. 9 and 10, respectively. ${ }^{1}$

The following is an approach to exploring cyclical behavior in the model. Suppose the externality parameter $\zeta$ increases. This causes the savings rate to increase. When consumers are willing to cut current consumption in exchange for higher future consumption, i.e. when intertemporal elasticity of substitution for consumption is high ( $\sigma$ is low), people start saving more. Hence there is a movement of labor from output production to human capital production. Human capital begins increasing. This implies faster accumulation of physical capital, if sufficient externality to human capital in production of physical capital is present. If people care about the future more (subjective discount rate $\rho$ is lower), consumption starts rising gradually with faster capital accumulation, leading to greater consumption-goods production in the future. This will eventually lead to a decline in savings rate. Two opposing effects come into play when savings rate is different from the equilibrium rate, causing a cyclical convergence to equilibrium. Hence, interaction between different parameters can cause cyclical convergence to equilibrium (Fig. 1) or may cause instability. For some parameter values we may have convergence to cycles (Fig. 2).

Using the numerical continuation package, Matcont, we further investigate the stability properties of cycles generated by different combinations of parameters. While some of the limit cycles generated by Andronov-Hopf bifurcation are stable (supercritical bifurcation), there could be some unstable limit cycles (subcritical bifurcation)

\footnotetext{
1 The graphs for transcritical bifurcation boundaries and Hopf bifurcation boundaries generated by varying different parameters can be found in the working paper version of the article at http://econpapers.repec.org/ paper/pramprapa/47231.htm.
} 


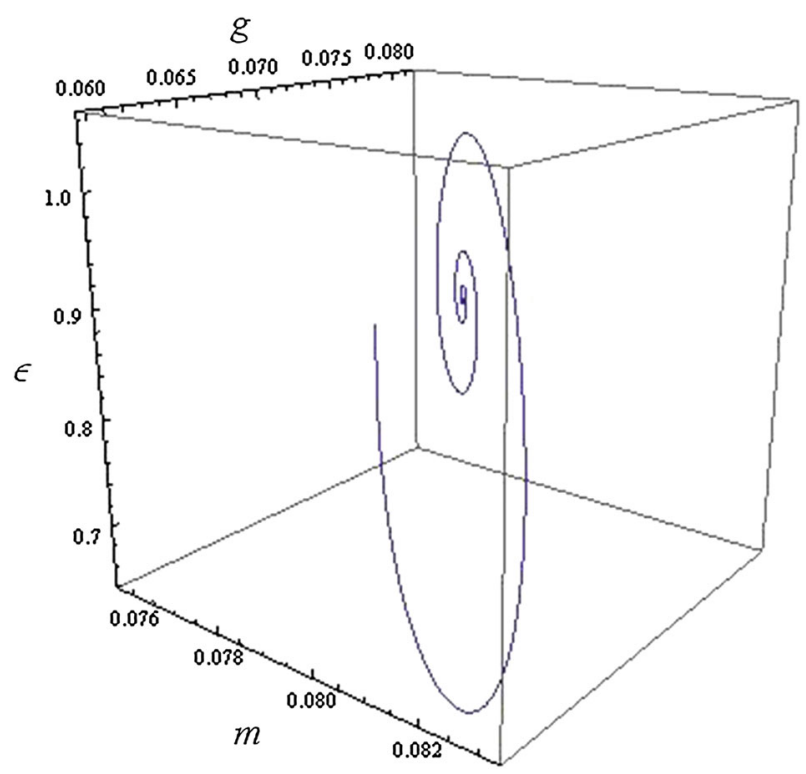

Fig. 1 Parameters in the stable region

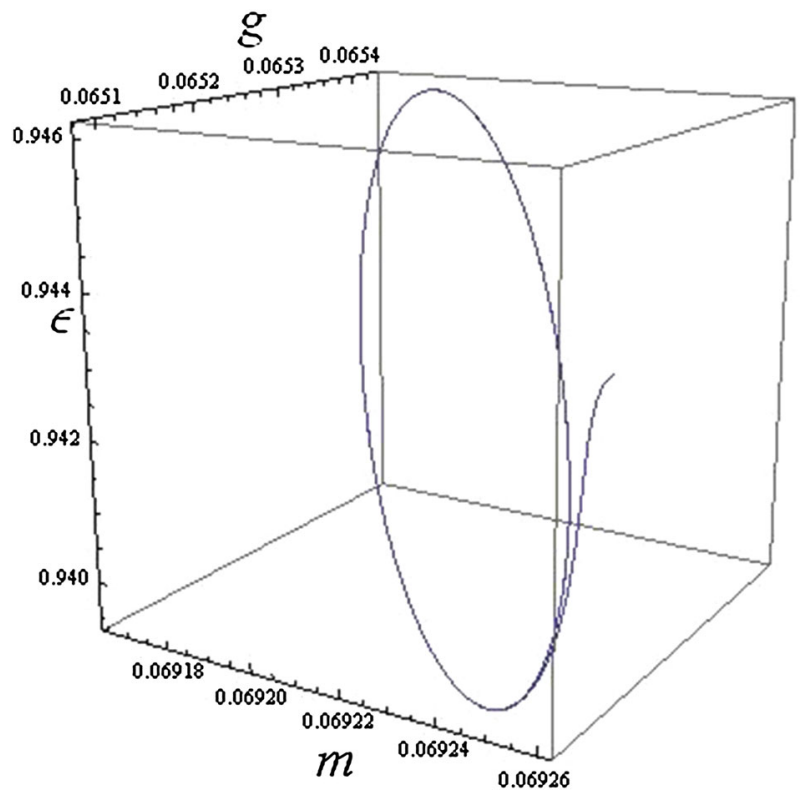

Fig. 2 Parameters on the Hopf bifurcation boundary

created as well. Table 1 reports the values of the share of capital $(\alpha)$, externality in production of human capital $(\zeta)$, and the inverse of intertemporal elasticity of substitution in consumption $(\sigma)$, at which Hopf bifurcations are generated when they are 
Table 1 Summary of the results

\begin{tabular}{|c|c|c|}
\hline Parameters & Equilibrium bifurcation & Bifurcation of limit cycle \\
\hline \multirow[t]{15}{*}{$\begin{array}{l}\alpha \text { (free) Other } \\
\text { parameters set at } \vartheta^{*}\end{array}$} & \multirow[t]{14}{*}{$\begin{array}{l}\text { Hopf }(\mathrm{H}) \text { : First Lyapunov coefficient } \\
\quad=0.00242, \alpha=0.738207\end{array}$} & $\begin{array}{l}\text { Limit point cycle }(\mathrm{LPC}) \text { : period }= \\
231.206, \alpha=0.7382042\end{array}$ \\
\hline & & Normal form coefficient $=0.007$ \\
\hline & & $\begin{array}{l}\text { Period doubling (PD): period }= \\
584.064, \alpha=0.7132369\end{array}$ \\
\hline & & Normal form coefficient $=0.910$ \\
\hline & & $\begin{array}{l}\text { Period doubling (PD): period }= \\
\quad 664.005, \alpha=0.7132002\end{array}$ \\
\hline & & Normal form coefficient $=-0.576$ \\
\hline & & $\begin{array}{l}\text { Period doubling }(\mathrm{PD}): \text { period }= \\
\quad 693.988, \alpha=0.7131958\end{array}$ \\
\hline & & Normal form coefficient $=-0.469$ \\
\hline & & $\begin{array}{l}\text { Period doubling (PD): period }= \\
\quad 713.978, \alpha=0.7131940\end{array}$ \\
\hline & & Normal form coefficient $=-0.368$ \\
\hline & & $\begin{array}{l}\text { Period doubling }(\mathrm{PD}) \text { : period }= \\
\quad 725.667, \alpha=0.7131932\end{array}$ \\
\hline & & Normal form coefficient $=-0.314$ \\
\hline & & $\begin{array}{l}\text { Period doubling (PD): period }= \\
\quad 784.104, \alpha=0.7131912\end{array}$ \\
\hline & & Normal form coefficient $=-0.119$ \\
\hline & \multicolumn{2}{|l|}{ Branch point (BP) } \\
\hline \multirow[t]{4}{*}{$\begin{array}{l}\zeta(\text { free }) \text { Other } \\
\text { parameters set at } \omega^{*}\end{array}$} & \multirow[t]{2}{*}{$\begin{array}{l}\text { Hopf }(\mathrm{H}) \text { : First Lyapunov coefficient } \\
\quad=0.00250, \zeta=0.107315\end{array}$} & $\begin{array}{l}\text { Limit point cycle (LPC): period } \\
=215.751, \zeta=0.1073147\end{array}$ \\
\hline & & Normal form coefficient $=0.009$ \\
\hline & \multirow{2}{*}{\multicolumn{2}{|c|}{$\begin{array}{l}\text { Hopf }(\mathrm{H}) \text { : First Lyapunov } \\
\text { coefficient }=0.00264, \zeta= \\
0.047059 \\
\text { Branch point: } \zeta=0.047059\end{array}$}} \\
\hline & & \\
\hline \multirow[t]{4}{*}{$\begin{array}{l}\zeta(\text { free }) \text { Other } \\
\text { parameters set at } \omega^{*}\end{array}$} & \multirow[t]{2}{*}{$\begin{array}{l}\text { Hopf }(\mathrm{H}) \text { : First Lyapunov coefficient } \\
\quad=0.00264, \sigma=0.278571\end{array}$} & $\begin{array}{l}\text { Limit point cycle }(\mathrm{LPC}) \text { : period }= \\
213.83, \sigma=0.1394026\end{array}$ \\
\hline & & Normal form coefficient $=0.009$ \\
\hline & $\begin{array}{l}\text { Hopf }(\mathrm{H}) \text { : First Lyapunov coefficient } \\
\quad=0.00249, \sigma=0.139394\end{array}$ & \\
\hline & Branch Point: $\sigma=0.278571$ & \\
\hline
\end{tabular}

treated as free parameters. ${ }^{2}$ A positive value of the first Lyapunov coefficient indicates creation of subcritical Hopf bifurcation. Thus, for each of the cases reported in Table 1, an unstable limit cycle (periodic orbit) bifurcates from the equilibrium. All of these cases also produce branch points (pitchfork/transcritical bifurcations).

2 The graphs of various codimension 1 and codimension 2 bifurcations generated by varying different parameters, the results of which are summarized in Table 1 can be found in the working paper version of the article at http://econpapers.repec.org/paper/pramprapa/47231.htm. 
Continuation of limit cycles from the Hopf point, when $\alpha$ is the free parameter, gives rise to limit point (Fold/saddle node) bifurcation of cycles. From the family of limit cycles bifurcating from the Hopf point, limit point cycle (LPC) is a fold bifurcation, where two limit cycles with different periods are present near the LPC point at $\alpha=0.738$. Continuing computation further from a Hopf point gives rise to a series of period-doubling (flip) bifurcations. Period-doubling bifurcation is defined as a situation in which a new limit cycle emerges from an existing limit cycle, and the period of the new limit cycle is twice that of the old one.

The first period-doubling bifurcation, at $\alpha=0.7132369$, has positive normal form coefficients, indicating existence of unstable double-period cycles. The rest of the period-doubling bifurcations have negative normal-form coefficients, giving rise to stable double-period cycles. The period of the cycle rapidly increases for very small perturbation in the parameter $\alpha$. The limit cycle approaches a global homoclinic orbit. A homoclinic orbit is a dynamical system trajectory, which joins a saddle equilibrium point to itself. In other words, a homoclinic orbit lies in the intersection of equilibrium's stable manifold and unstable manifold. There exists the possibility of reaching chaotic dynamics in the decentralized version of Uzawa-Lucas model through a series of period-doubling bifurcations. For the cases in which $\zeta$ and $\sigma$. are free parameters, we carry out the continuation of limit cycle from the first Hopf point. Both cases give rise to limit point cycles with a nonzero normal-form coefficient, indicating the limit cycle manifold has a fold at the LPC point. Similar results are found, if we carry out the continuation of limit cycles from the second Hopf point for each of these cases.

\section{Conclusion}

Transcritical bifurcation and Hopf bifurcation boundaries, corresponding to different combinations of parameters, are located for the decentralized version of the Uzawa-Lucas model. Examination of the stability properties of the limit cycles generated from various Hopf bifurcations in the model depicts occurrence of limit point-of-cycles bifurcations and period-doubling bifurcations within the model's feasible parameter set. The series of period-doubling bifurcations confirms the presence of global bifurcation. Period-doubling bifurcations also reveal the possibility of chaotic dynamics, occurring in the converged limit of the sequence of period doubling. In contrast, the centralized social planner solution is always saddle path stable, with no possibility of bifurcation within the feasible parameter set, but with no decentralized informational privacy. Thus, the externality of the human capital parameter plays an important role in determining the dynamics of the decentralized Uzawa-Lucas model. Our result emphasizes the need for simulations of decentralized macro econometric models at settings throughout the parameter estimates' confidence regions, rather than at the point estimates alone, since dynamical inferences otherwise can produce oversimplified conclusions subject to robustness problems. 


\section{Appendix 1}

In the steady state, the constancy of $m, g$, and $\varepsilon$ implies $\frac{\dot{K}}{K}=\frac{\dot{C}}{C}=\frac{\dot{Y}}{Y}=\frac{\dot{k}}{k}+n=$ $\frac{(1-\alpha+\zeta)}{(1-\alpha)} \eta(1-\varepsilon)+n$

Transversality conditions require $\lim _{t \rightarrow \infty} \lambda_{t} K_{t}=0$ and $\lim _{t \rightarrow \infty} \mu_{t} h_{t}=0$, which in turn imply $\rho>(1-\sigma) \frac{(1-\alpha+\zeta)}{(1-\alpha)} \eta(1-\varepsilon)$, where $\lambda$ and $\mu$ are costate variables (Appendices 2 and 3).

\section{Appendix 2}

Social planner problem: $\mathcal{H}=\frac{\left[c(\tau)^{1-\sigma}-1\right]}{(1-\sigma)}+\lambda\left[A k^{\alpha} \varepsilon^{1-\alpha} h^{1-\alpha+\zeta}-c-(n+\delta) K\right]+$ $\mu[\eta h(1-\varepsilon)]$

Lucas (1988) showed that the first-order conditions are

(1) $c: c^{-\sigma} e^{-(\rho-n)}=\lambda$,

(2) $\varepsilon: \lambda(1-\alpha) A k^{\alpha} \varepsilon^{-\alpha} h^{1-\alpha+\zeta}=\mu \eta h$,

(3) $k: \lambda\left[\alpha A k^{\alpha-1} \varepsilon^{1-\alpha} h^{1-\alpha+\zeta}-(n+\delta)\right]=-\lambda^{\prime \prime}$

(4) $h: \lambda(1-\alpha+\zeta) A k^{\alpha} \varepsilon^{1-\alpha} h^{-\alpha+\zeta}+\mu \eta(1-\varepsilon)=-\mu^{\prime \prime}$.

\section{Appendix 3}

Decentralized or market solution:

$$
\mathcal{H}=\frac{\left[c(\tau)^{1-\sigma}-1\right]}{(1-\sigma)}+\lambda\left[A k^{\alpha} \varepsilon^{1-\alpha} h^{1-\alpha} h_{a}^{\zeta}-c-(n+\delta) K\right]+\mu[\eta h(1-\varepsilon)]
$$

Lucas (1988) showed that the first-order conditions are

(1) $c: c^{-\sigma} e^{-(\rho-n)}=\lambda$,

(2) $\varepsilon: \lambda(1-\alpha) A k^{\alpha} \varepsilon^{-\alpha} h^{1-\alpha} h_{a}^{\zeta}=\mu \eta h$,

(3) $k: \lambda\left[\alpha A k^{\alpha-1} \varepsilon^{1-\alpha} h^{1-\alpha} h_{a}^{\zeta}-(n+\delta)\right]=-\dot{\lambda}$,

(4) $h: \lambda(1-\alpha) A k^{\alpha} \varepsilon^{1-\alpha} h^{-\alpha} h_{a}^{\zeta}+\mu \eta(1-\varepsilon)=-\dot{\mu}$.

\section{References}

Arnold, L.G.: Stability of the market equilibrium in Romer's model of endogenous technological change: a complete characterization. J. Macroecon. 22, 69-84 (2000a)

Arnold, L.G.: Endogenous technological change: a note on stability. Econ. Theory 16, 219-226 (2000b)

Arnold, L.G.: The dynamics of jones R\&D model. Rev. Econ. Dyn. 9, 143-152 (2006)

Banerjee, S., Barnett, W.A., Duzhak, E.A., Gopalan, R.: Bifurcation analysis of Zellner's marshallian macroeconomic model. J. Econ. Dyn. Control 35, 1577-1585 (2011)

Barnett, W.A., Duzhak, E.A.: Empirical assessment of bifurcation regions within new Keynesian models. Econ. Theory 45, 99-128 (2010) 
Barnett, W.A., Ghosh, T.: Bifurcation analysis of an endogenous growth model. J. Econ. Asymmetries 10, 53-64 (2013)

Barnett, W.A., He, Y.: Stability analysis of continuous-time macroeconometric systems. Stud. Nonlinear Dyn. Econ. 3, 169-188 (1999)

Barnett, W.A., He, Y.: Stabilization policy as bifurcation selection: would stabilization policy work if the economy really were unstable? Macroecon. Dyn. 6, 713-747 (2002)

Barnett, W.A., He, Y.: Existence of singularity bifurcation in an Euler-equations model of the United States economy: grandmont was right. Phys. A 387, 3817-3825 (2008)

Barnett, W.A., Eryilmaz, U.: Hopf bifurcation in the clarida, gali, and gertler model. Econ. Model. 31, 401-404 (2013a)

Barnett, W. A., Eryilmaz, U.: An analytical and numerical search for bifurcations in open economy new Keynesian models. Macroecon. Dyn., forthcoming (2013b)

Barro, R. J., Sala-i-Martin, X.: Economic Growth. The MIT Press, Second Edition (2003)

Benhabib, J., Perli, R.: Uniqueness and indeterminacy on the dynamics of endogenous growth. J. Econ. Theory 63, 113-142 (1994)

Guckenheimer, J., Holmes, P.: Nonlinear oscillations, dynamical systems, and bifurcations of vector fields. Springer, New York (1983)

Guckenheimer, J., Myers, M., Sturmfels, B.: Computing Hopf bifurcations, I. SIAM J. Numer. Anal. 34, 1-21 (1997)

Grossman, G., Helpman, E.: Endogenous product cycles. Econ. J. 101, 1229-1241 (1991b)

Jones, C.I.: R\&D-based models economic growth in a world of ideas. J. Polit. Econ. 103, 759-784 (1995)

Jones, C. I.: Sources of U.S. economic growth in a world of ideas. Am. Econ. Rev. 220-239 (2002)

Kuznetsov, YuA: Elements of applied bifurcation theory, 2nd edn. Springer, New York (1998)

Leeper, E., Sims, C.: Toward a modern macro model usable for policy analysis'. NBER Macroeconomics Annual, 81-117 (1994)

Lucas, R.E.: On the mechanics of economic development. J. Monet. Econ. 22, 3-42 (1988)

Mondal, D.: Stability analysis of the Grossman-Helpman model of endogenous product cycles. J. Macroecon. 30, 1302-1322 (2008)

Mattana, P.: The Uzawa-Lucas endogenous growth model, Ashgate Publishing Ltd. (2004)

Romer, P.M.: Endogenous technological change. J. Polit. Econ. 98, S71-S102 (1990)

Uzawa, H.: Optimum technical change in an aggregative model of economic growth. Int. Econ. Rev. 6, 18-31 (1965) 